\title{
Adult accompaniment and the risk of pedestrian injury on the school-home journey
}

\author{
Ian Roberts
}

\begin{abstract}
Study objective-To quantify the effect of adult accompaniment on the risk of pedestrian injury on the school-home journey.
\end{abstract}

Design-A community based casecontrol study.

Setting-The Auckland region of New Zealand.

Participants-Cases $(n=54)$ were all children killed or hospitalized as a result of a pedestrian injury occurring on the school-home journey between 1 January 1992 and 1 March 1994. The response rate for the case group was $98 \%$. Controls $(n=157)$ were a random sample of all children who walk to and from school in the study region. The response rate for the control group was $100 \%$.

Main results-Adult accompaniment on the school-home journey was associated with a reduced risk of injury (odds ratio (OR) $0.36,95 \%$ confidence interval (CI) 0.04 to 1.66$)$. This effect persisted after controlling for age, sex, and socioeconomic status (OR 0.31, 95\% CI 0.07 to 1.49).

Conclusions-Adult accompaniment on the school-home journey may have the potential to significantly reduce child pedestrian injury rates. The effect of adult accompaniment may have important implications for the interpretation of child pedestrian exposure studies.

(Injury Prevention 1995; 1: 242-244)

Keywords: epidemiological methods, traffic accidents, case-control study.

Pedestrian injuries are a leading cause of death and serious injury for school aged children. ${ }^{1} \mathrm{~A}$ large proportion of these injuries occur while children are walking to or from school. ${ }^{2}$ Exposure studies have demonstrated large differences in adult accompaniment on the school-home journey. However, the effect of such adult accompaniment on the risk of child pedestrian injury has never been evaluated in an etiologic epidemiologic study. ${ }^{3}$ We used data from a large scale population based casecontrol study to quantify the effect of adult accompaniment on the risk of pedestrian injury occurring on the school-home journey.

\section{Methods}

The study base comprised all children between the ages of 5 and 15 years, resident within the study region, who walked to and from school, during the period 1 January 1992 through 1 March 1994. At the time of the 1991 census, the Auckland region had a predominantly urban population of 936981 of whom approximately 133848 were aged between 5 and 15 years. ${ }^{4}$

Study cases were all children aged 5 to 15 years who were admitted to hospital or killed as a result of a pedestrian-motor vehicle collision occurring on the school-home journey. Children resident outside of the Auckland region were excluded from the case group. Fatal cases were identified by regular surveillance of the records of the coroner's pathologist. In Auckland, all children whose deaths have resulted from injury are subject to a coroner's postmortem. Hospitalized cases were identified by regular surveillance at both of the hospitals in the region that admit injured children.

Each control was selected by, first, randomly selecting a school from a list of all schools in the study region, with a sampling probability in proportion to the number of children on the school roll. Each selected school was then visited by the study staff who randomly selected one child from the school roll. To reduce the possibility of severe loss of efficiency due to substantial age and sex differences between cases and controls, controls were frequency matched to cases by age and sex.

After each subject was identified, an interview was scheduled with the parents. Parents of hospitalized cases were interviewed in hospital, at a time determined in consultation with the hospital staff. The parents of fatal cases and parents of controls were interviewed in their own homes. Parents were asked to complete an interviewer administered questionnaire that included questions about sociodemographic characteristics and details of their child's pedestrian travel on the school-home journey. Parents of cases were asked to provide details of the child's pedestrian travel for the day of injury, whereas parents of controls were asked about travel for the most recent full day attended. If, on this day, the selected child did not walk, that child was excluded from the control group. Parents of cases and controls were also asked whether the child was accompanied, and if so, by whom.

Socioeconomic position was classified according to the New Zealand based Elley Irving social class classifications. ${ }^{5}$ Both maternal and paternal occupations were classified, with the highest of the two being taken as the socioeconomic variable for each child. For the purpose of analysis three ethnic groups were constructed: Maori, Pacific Islander (Samoan, Tongan, Cook Island, Fijian, Niuean), and other (European, Chinese, Indian, other). 
Relative risks were estimated by calculation of odds ratios (ORs). The univariate ORs have $95 \%$ confidence intervals (CIs) calculated by the method of Cornfield except when cell numbers are small when the exact method was used. Multivariate ORs were obtained from unconditional logistic regression, as were their CIs. Because the current analyses are based on a subset of cases and controls in the main study (children aged 5-15 years who walk to school) the ratio of controls to cases is not an exact multiple. However, this has no implications for validity. The study was approved by the University of Auckland human subjects ethics committee.

\section{Results}

A total of 170 injured child pedestrians aged between 5 and 15 years were identified over the study period, $167(98 \%)$ of whom agreed to participate. Of these, $54(32 \%)$ were injured while walking to or from school. Eighteen children were injured on the journey to school and 36 were injured on the journey home from school. Thirty five $(65 \%)$ of the cases were aged between 5 and 9 years, and 25 of these children were injured on the way home from school. Nineteen $(35 \%)$ of the cases were aged between 10 and 14 years, 11 of whom were injured on the way home from school. Three hundred twenty control children between the ages of 5 and 15 years were identified, $157(49 \%)$ of whom walked to and from school on the most recent full school day. The response rate for the controls was $100 \%$.

The table shows the numbers and ORs for the variables of interest. The control group for these analyses comprised walking children only. Estimates based on unconditional logistic regression, with the matching factors age and sex as confounding variables, were conducted but this had little effect on the OR estimates and therefore are not presented. Because the majority of injuries occurred on the homeward bound journey, the exposure distribution for controls was based on that journey. For the calculation of multivariate ORs the variables age, sex, and socioeconomic status were included in the model. In the multivariate analyses, adult accompaniment was associated with a greatly reduced risk of injury (OR 0.31 , $95 \%$ CI 0.07 to 1.49 ). When socioeconomic status was replaced by ethnic group in the model, the coefficients were essentially unchanged. When the analyses were repeated excluding the 18 cases injured on the journey to school, the univariate OR associated with adult accompaniment was 0.27 (95\% CI 0.01 to 1.89$)$ and the multivariate $\mathrm{OR}$ was $0 \cdot 18(95 \% \mathrm{CI} 0.02$ to $1 \cdot 54)$.

\section{Discussion}

This study aimed to quantify the effect of adult accompaniment on the risk of pedestrian injury on the school-home journey. Ours is the first analytic epidemiologic study to examine this question. The results suggest that adult accom-
Risk factors for pedestrian injuries on the school-home journey

\begin{tabular}{|c|c|c|c|}
\hline Variable & $\begin{array}{l}\text { Cases } \\
(n=54)\end{array}$ & $\begin{array}{l}\text { Controls } \\
(n=157)\end{array}$ & $\begin{array}{l}\text { Crude OR } \\
(95 \% \text { CI })\end{array}$ \\
\hline \multicolumn{4}{|c|}{ Accompanied by an adult } \\
\hline $\begin{array}{l}\text { Yes } \\
\text { No }\end{array}$ & & $\begin{array}{l}15 \\
142\end{array}$ & $\begin{array}{l}0.36(0.04 \text { to } 1.66)^{\star} \\
1.00\end{array}$ \\
\hline \multicolumn{4}{|c|}{ Socioeconomic position } \\
\hline $\begin{array}{l}\text { I, II, III } \\
\text { IV, V } \\
\text { VI and others }\end{array}$ & $\begin{array}{l}7 \\
23 \\
24\end{array}$ & $\begin{array}{l}26 \\
71 \\
60\end{array}$ & $\begin{array}{l}1.00 \\
1.20(0.42 \text { to } 3.51) \\
1.49(0.52 \text { to } 4.35)\end{array}$ \\
\hline \multicolumn{4}{|l|}{ Ethnic group } \\
\hline Maori & 10 & 43 & $0.70(0.28$ to 1.72$)$ \\
\hline Pacific Islander & 21 & 45 & $1.40(0.66$ to 2.99$)$ \\
\hline Other & 23 & 69 & 1.00 \\
\hline
\end{tabular}

$\star$ Exact CI.

paniment may be associated with a considerable reduction in risk.

Although an attempt was made to ascertain all child pedestrian injuries resulting in death or hospitalization arising in the study base, some events may have been missed. Nevertheless, provided that case identification was independent of risk factor status no bias would have been introduced. As there is no reason to suspect this occurred, incomplete ascertainment of cases does not present a threat to validity. Similarly, given the high response rates for cases and controls, the potential for bias due to non-response is also minimal.

The sampling frame for control children was all schoolchildren in the study region. With the possible exception of children from itinerant families, this sampling frame can be expected to have included all schoolchildren who walk to school in the study base. Although this method of sampling was relatively cost inefficient, given the large number of controls who were discarded, it is likely to have provided a valid estimate of the exposure distribution in the study base.

The risk to children who were accompanied by an adult was less than half that of children who were not accompanied. Because there were very few accompanied cases, however, the precision of the effect estimate is low (wide CIs). However, it is important not to confuse the lack of precision as equivalent to the absence of a protective effect. Indeed, the results are suggestive of a strong protective effect of accompaniment.

Lack of power is a problem in many epidemiologic studies of injury, because many of the risk factors of interest will be etiologically relevant only in certain scenarios. ${ }^{6} \mathrm{~A}$ combination of etiologically unrelated outcomes would result in narrower confidence intervals but at the expense of bias. In this study combining injuries occurring on the journey to and from school appears to have resulted in an underestimation of the protective effect of adult accompaniment, because the exposure information for controls was based on accompaniment for the journey home. When the analyses were restricted to injuries occurring on the journey home from school the protective effect was stronger but less precise. This finding provides further indirect support for a genuine protective effect of adult accompaniment. 
IMPLICATIONS FOR PREVENTION

If these results were confirmed in other studies, the reduced risk associated with adult accompaniment would have important implications for prevention. It would suggest that strategies that facilitate adult accompaniment would greatly reduce injury rates. Such strategies might include more flexible working hours, changes in the timing of the school day, or after school care for children of working parents. It would also have important implications for the interpretation of pedestrian exposure studies. In particular, the need to take adult accompaniment into account when attempting to explain differences in injury rates on the basis of exposure.
This study was funded by the Health Research Council of New Zealand.

Rivara FP. Child pedestrian injuries in the United States. $A m$ f Dis Child 1990; 144: 692-6.

2 Joly MF, Foggin PM, Pless IB. Geographical and socioecological variations of traffic accidents among children. Soc Sci Med 1991; 33: 765-9.

3 Roberts I, Norton R. Auckland children's exposure to risk as pedestrians. N $Z$ Med f 1994; 107: 331-3.

4 Department of Statistics. Consensus of population and dwellings, March 1991. Wellington: Department of Statistics, 1991.

5 Elley WB, Irving JC. The Elley Irving socioeconomic index 1981 census revision. NZ $\mathcal{f}$ Educ Studies 1985; 20 115-28.

6 Roberts I. Methodologic issues in injury case-control studies. Injury Prevention 1995; 1: 45-8.

\section{Daytime running lights}

On a recent trip to the US I realized that the message about the effectiveness of daytime running lights has not sunk in. A recent study from the Road Safety Directorate of Transport Canada, however, emphasizes the importance of this measure: their use reduced opposite direction two vehicle collisions by $8.3 \%$. My old car does not put these lights on automatically; I have to turn a switch! Most recent US cars do turn them on so drivers have to turn the switch in the opposite direction to diminish their visual contrast. How strange.

\section{More biters}

In addition to the report from Greece about biting monkeys, readers are also reminded that grey squirrels (an American import to Europe) are also biters: a report in the $B M \mathcal{F}$ (December 1994) states that 24 of 2591 animal bites treated in two accident and emergency departments in Edinburgh were by squirrels 'biting the hand that was feeding them'. 